\title{
Os prejuízos funcionais de pessoas com transtorno de ansiedade social: uma revisão*
}

\author{
Functional impairment of persons with social anxiety disorder: a review
}

\author{
Luciene Vaccaro de Morais'; J osé Alexandre S. Crippa'"; Sonia Regina Loureiro'II \\ 'Mestrado. Terapeuta ocupacional, Serviço de Terapia Ocupacional, Hospital das Clínicas, \\ Faculdade de Medicina de Ribeirão Preto, Universidade de São Paulo (USP), Ribeirão Preto, SP. \\ "Doutor. Professor, Departamento de Neurologia, Psiquiatria e Psicologia Médica, Faculdade de \\ Medicina de Ribeirão Preto, USP. \\ IIIDoutora. Professora, Departamento de Neurologia Psiquiatria e Psicologia Médica, Faculdade \\ de Medicina de Ribeirão Preto, USP.
}

Endereço para correspondência

\begin{abstract}
RESUMO
O transtorno de ansiedade social caracteriza-se pelo medo acentuado e persistente de situações sociais ou de desempenho, podendo influenciar a vida cotidiana dos portadores. Objetiva-se identificar, na literatura indexada, estudos empíricos com instrumentos de avaliação que abordem os prejuízos funcionais experimentados por pessoas com transtorno de ansiedade social no desempenho de atividades cotidianas e analisar as implicações de tais dificuldades. Procedeu-se a uma busca sistemática junto às bases de dados indexadas, identificando dez artigos, que foram agrupados em dois conjuntos: a) os relativos à proposição de instrumentos de avaliação e análise das qualidades psicométricas $(n=4)$; e b) os relativos ao impacto do transtorno de ansiedade social para a qualidade de vida e para as atividades cotidianas $(n=6)$. Os estudos psicométricos ressaltaram a pouca atenção dada a instrumentos validados para avaliar especificamente os prejuízos funcionais. Os trabalhos relativos ao impacto na qualidade de vida e nas atividades cotidianas constataram a presença de insatisfação com a vida e com a saúde em pacientes com transtorno de ansiedade social. A análise da literatura apontou para a importância da avaliação dos prejuízos funcionais do transtorno de ansiedade social no funcionamento cotidiano, por meio de medidas objetivas. Destaca-se a relevância de tais avaliações no planejamento e na intervenção de atividades voltadas ao transtorno de ansiedade social no contexto das práticas multidisciplinares de saúde mental.
\end{abstract}

Descritores: Ansiedade social, prejuízos funcionais, instrumentos, escalas, revisão. 


\section{ABSTRACT}

Social anxiety disorder is characterized by strong and persistent fear of social situations, impairing the daily life of affected people. The objective of the present work was to identify empirical studies involving instruments that assess the functional impairment experienced by persons with social anxiety disorder in the execution of daily activities and to analyze the implications of such difficulties. A systematic search of indexed databases was performed. Ten articles were identified and assigned to two groups: a) papers dealing with the proposal of instruments for the evaluation and analysis of psychometric qualities $(n=4)$; and b) papers dealing with the impact of social anxiety disorder on the quality of life and on daily activities ( $n$ $=6$ ). The psychometric studies emphasized the little attention paid to instruments validated in order to specifically evaluate functional impairment, and the papers dealing with the impact on quality of life and on daily activities detected the presence of dissatisfaction with life and health in patients with social anxiety disorder. Analysis of the literature stressed the importance of evaluating the functional impairment caused by social anxiety disorder in daily functioning using objective measurements. The relevance of such evaluations for planning and interventions focusing on social anxiety disorder in the context of multidisciplinary mental health practice is highlighted.

Keywords: Social anxiety, functional impairment, instruments, scales, review.

\section{NTRODUÇÃO}

O transtorno de ansiedade social (TAS), também conhecido como fobia social, é um dos transtornos de ansiedade mais comuns e tem sido considerado um grave problema de saúde pública, chamando a atenção pela alta prevalência, estimada entre 2,6 e 16\%, dependendo do critério diagnóstico adotado. No entanto, tal condição clínica, por vezes, é sub-reconhecida e subdiagnosticada por estar freqüentemente acompanhada por comorbidades como depressão, distimia, abuso e dependência de álcool e drogas, ideação suicida, transtornos alimentares e outros tipos de transtornos de ansiedade ${ }^{1-7}$.

O TAS é caracterizado pelo medo acentuado e persistente de situações sociais ou de desempenho em tarefas frente às quais o indivíduo sente vergonha e temor de exposição, experimentando invariavelmente uma resposta imediata de ansiedade. Os principais medos relacionados à exposição são: a) parecer ridículo; b) dizer tolices; c) ser observado pelas outras pessoas; d) interagir com estranhos ou pessoas do sexo oposto; e) ser o centro das atenções; f) comer, beber ou escrever em público; g) falar ao telefone; e h) usar banheiros públicos. Quando tais situações não podem ser evitadas, são vivenciadas com grande ansiedade e, na maioria das vezes, acompanhadas por sinais e sintomas como: palpitações, tremores, sudorese, desconforto gastrointestinal, tensão muscular, rubor facial e confusão ${ }^{2,8}$.

Frente às situações percebidas como ameaçadoras, observa-se uma tendência à evitação de tais eventos, o que na vida cotidiana pode se caracterizar pela recusa em apresentar trabalhos ou seminários, escrever, comer e falar ao telefone na frente de colegas, realizar entrevistas ou conversas na escola, com professores, ou no trabalho, com chefes, iniciar uma relação afetiva, viajar com os amigos, participar de jogos e de atividades esportivas, entre outras. Considera-se, assim, que os prejuízos relacionados ao TAS incidem diretamente na vida cotidiana das pessoas, possivelmente prejudicando a sua qualidade de vida ${ }^{9}$.

Problemas como o distanciamento das relações pessoais, as dificuldades na participação em atividades profissionais, sociais, de lazer e de autocuidado, assim como as restrições de contatos e atividades, constituem-se em prejuízos funcionais que interferem nas ocupações diárias e na 
participação na vida cotidiana, com implicações para as condições de saúde do indivíduo.

A presença de prejuízos funcionais ou de incapacidades é comumente referida, nos estudos sobre o TAS, como uma forma de manifestação dos prejuízos associados ao transtorno. Alguns estudos $^{5,10,11}$, ao referirem os prejuízos funcionais, o fazem caracterizando o impacto do transtorno nas atividades habituais do dia-a-dia.

A Classificação Internacional de Funcionalidade, Incapacidade e Saúde (CIF), de maneira similar, apresenta incapacidade como um termo genérico para caracterizar deficiências, limitações de atividades e restrições de participação em diferentes domínios, englobando a interação entre o indivíduo e seus fatores contextuais ${ }^{12}$.

Para as pessoas com TAS, os prejuízos funcionais se configuram como um ponto central de suas dificuldades, sendo necessário o reconhecimento dos indicadores que os caracterizam, de modo a instrumentar o planejamento e a aplicação de práticas de saúde ${ }^{7,10,11}$.

Dado o impacto das dificuldades vivenciadas na vida cotidiana para os portadores do TAS, em geral pessoas jovens, em etapa de importantes aquisições escolares e profissionais, considera-se como relevantes intervenções de saúde mental que tenham a finalidade de minimizar tais prejuízos. Para tal, as escalas de avaliação, por fornecerem medidas aferidas e sistemáticas, têm uma relevante contribuição, por permitirem a comparação de grupos contrastante e das mesmas pessoas em momentos diversos, em pesquisas de intervenção.

Considera-se que a caracterização dos indicadores de prejuízos funcionais carece de maior precisão.

Frente a isso, objetivou-se identificar, na literatura indexada, estudos empíricos com instrumentos de avaliação que abordem os prejuízos funcionais experimentados por pessoas com TAS, na participação de atividades cotidianas, analisando-se as implicações de tais dificuldades.

Procedimento

Procedeu-se a uma busca sistemática junto às bases de dados indexadas MEDLINE, LI LACS e SciELO, compreendendo o período de 1999 a 2007, complementada pela identificação de artigos presentes nas referências bibliográficas dos estudos selecionados.

Para tal, utilizou-se as seguintes palavras-chave: dificuldade cotidiana, prejuízo e funcionamento psicossocial, cruzadas com fobia social, ansiedade social e seus correspondentes em inglês: daily disability, impairment e psychosocial functioning.

Adotou-se como critérios de exclusão estudos relacionados a: a) avaliação dos aspectos clínicos do TAS; b) revisão da literatura; c) diagnóstico diferencial e comorbidades; d) tratamentos psiquiátricos e psicológicos do TAS.

Tomou-se como critérios de inclusão os estudos: a) escritos em idioma inglês, português, francês e espanhol; b) com amostras de adolescentes e adultos; c) envolvendo instrumentos de avaliação que abordam aspectos psicossociais associados ao TAS; e d) visando à avaliação do impacto do TAS na qualidade de vida e nos afazeres cotidianos.

Inicialmente, foram identificados 45 artigos e, após a aplicação dos critérios de inclusão e exclusão, foram excluídos 35 artigos, por abordarem aspectos descritivos, epidemiológicos e clínicos relativos a sinais e sintomas de medo, ansiedade e do comportamento de esquiva frente às situações de interação ou de desempenho, ou ainda por tratarem de intervenções farmacológicas no TAS. Foram selecionados, assim, 10 artigos que serão objetos de análise. 


\section{RESULTADOS}

Para a análise dos estudos, os mesmos foram agrupados em dois conjuntos: a) os relativos à proposição de instrumentos de avaliação e análise das suas qualidades psicométricas $(n=4)$; e b) os relativos ao impacto do TAS na qualidade de vida e nas atividades cotidianas $(n=6)$.

Características psicométricas de instrumentos de avaliação dos prejuízos funcionais associados ao TAS

Foram incluídos, neste conjunto, quatro estudos ${ }^{10,11,13,14}$.

Com relação aos delineamentos, as amostras dos estudos variaram de 46 a 360 sujeitos ( mediana $=203$ ) de ambos os sexos, na faixa etária dos 15 aos 65 anos. Os estudos avaliaram amostras clínicas compostas por indivíduos com TAS, comparativamente a outros sujeitos com outros tipos de transtornos de ansiedade e a controles saudáveis, sistematicamente avaliados quanto à ausência de comorbidades psiquiátricas. Para a inclusão nos estudos, os participantes foram avaliados por meio de entrevistas estruturadas, fundamentadas nos critérios diagnósticos do Manual de Diagnóstico e Estatística das Perturbações Mentais, 3a edição revisada e 4ạ edição (DSM-III-R e DSM-IV) como: Anxiety Disorders Interview Schedule for DSM-IV - Lifetime version (ADIS-IV-L) e a Structured Clinical Interview (SCID). Dentre os quatro artigos analisados, em um deles ${ }^{11}$ foi relatado o treinamento dos entrevistadores, profissionais qualificados e a sistematização da aplicação das entrevistas diagnósticas. Em todos os estudos, foram adotados como critérios de exclusão a presença de comorbidades psiquiátricas, tais como: transtorno bipolar, transtornos psicóticos, abuso ou dependência de substâncias nos últimos 3 meses e depressão maior.

A avaliação específica dos prejuízos funcionais associados ao TAS teve como referência básica os dois instrumentos propostos por Liebowitz, a saber: Liebowitz Self Rating Disability Scale (LSRDS) e Disability Profile/Clinician Rated (DP). Tais escalas foram avaliadas quanto às qualidades psicométricas por um grupo de pesquisadores, incluindo o próprio Liebowitz ${ }^{10}$.

No referido estudo ${ }^{10}$, os autores realizaram avaliação da validade concorrente da LSRDS e da DP com a Sheehan Disability Scale (SDS) e a Liebowitz Panic and Social Phobic Disorder-Severity of Illness Rating Form Anxiety Scale (LPSPD-S), avaliando um grupo de pessoas com fobia social e pessoas sem desordens psiquiátricas. Para tal, foram avaliados 32 pacientes com TAS e 14 sujeitos sem transtorno psiquiátrico. Constataram que os instrumentos LSRDS e DP avaliaram as incapacidades relacionadas ao TAS de forma consistente e correta quando comparados à avaliação com a Clinical Global Impressions-Severity of Illness Scale (CGI-S), a Liebowitz Social Anxiety Scale (LSAS) e a LPSPD-S. Tal investigação mostrou que os portadores de TAS se avaliaram e foram avaliados por profissionais clínicos tendo prejuízos de moderado a grave nas áreas referentes à escola, trabalho, relações familiares, namoro/casamento, amizades/rede social e outros interesses. Os indicadores de prejuízos funcionais, nesse estudo, abordaram contextos de desempenho relacionados à escola e ao trabalho e contextos de interação e de relacionamento.

Em relação às qualidades psicométricas, os instrumentos tiveram boa consistência interna, sendo que a DP apresentou um alfa de Cronbach de 0,87 para a avaliação relativa às "duas últimas semanas" e de 0,90 para a avaliação do "pior momento ao longo da vida". A LSRDS apresentou alfa de Cronbach de 0,92 para a avaliação dos dois momentos. Quanto à validade concomitante, as subescalas mostraram correlação significativa com a SDS, sendo a variação do coeficiente de correlação $(r)$ de 0,56 a 0,79 para a DP e de 0,56 a 0,73 para a LSRDS, sugerindo 
que as escalas foram similares à SDS quanto à capacidade de avaliar prejuízos funcionais ${ }^{10}$.

Com relação à validade de critério, as escalas mostraram significativa diferença entre os resultados obtidos por pessoas com e sem TAS, apontando para a capacidade de detectar os prejuízos associados à fobia social de forma diferenciada dos outros prejuízos associados a ansiedade social e evitação ${ }^{10}$.

No segundo estudo analisado ${ }^{11}$, os autores, tomando como pressuposto que nos estudos sobre transtornos de ansiedade os construtos "qualidade de vida" e "prejuízos funcionais" são fortemente relacionados e comumente usados como sinônimos, objetivaram verificar se estes construtos devem ser avaliados como uma única dimensão ou se requerem avaliação separada. Para tal, avaliaram 96 pessoas com diagnóstico de TAS por meio da Entrevista Clínica Estruturada para o DSM-IV (SCID-IV). Analisaram a correlação entre medidas de qualidade de vida por meio do Quality of Life Inventory (QOLI), medidas de avaliação de sintomatologia com a Social Interaction Anxiety Scale (SIAS) e medidas de prejuízos funcionais por meio da SDS, LSRDS e da DP.

Os autores procederam à correlação entre as medidas de prejuízos funcionais, sintomatologia e qualidade de vida, destacando que as medidas de prejuízos funcionais mostraram-se fortemente relacionadas. Tendo em vista a alta correlação entre tais medidas, os autores procederam à análise de regressão conduzida por meio das medidas de sintomatologia, de qualidade de vida e de prejuízos funcionais. Nesse estudo, verificaram que as medidas de prejuízos funcionais e de sintomatologia apresentaram áreas de sobreposição na relação com a medida de qualidade de vida, embora a relação entre estas medidas tenha sido distinta quanto ao grau de significância.

Com base nestes estudos, os autores mostraram que as medidas de prejuízos funcionais e de qualidade de vida descreveram fenômenos distintos, podendo ser observado que quanto maior a intensidade de sintomas relacionados ao TAS, maiores os prejuízos funcionais e menor a qualidade de vida. Os prejuízos funcionais, tais como abordados nesse estudo, podem ser entendidos como mediadores da expressão dos sintomas e da percepção da qualidade de vida.

O terceiro estudo analisado ${ }^{13}$ investigou as propriedades psicométricas de escalas de avaliação de prejuízos associados aos transtornos de ansiedade, usando o modelo da equação estrutural para detectar quais as variáveis necessárias para conhecer e interpretar os prejuízos. Para tanto, avaliaram 360 pessoas portadoras de transtorno de ansiedade, por meio da SCID-IV, do DSMIII-R (Global Assessment of Functioning - GAF) e dos seguintes instrumentos: SDS, Social Adjustment Scale-Self Report (SAS-S), LSRDS, Medical Outcome Study Short Form (MOS), Beck Depression Inventory (BDI), State-Trait Anxiety Inventory (STAI) e DP. Nesse estudo, oS prejuízos funcionais foram identificados pelo desempenho no trabalho, nos relacionamentos e nas atividades fora do trabalho como as principais dimensões a serem avaliadas.

Ao analisar a contribuição de cada um dos instrumentos para o modelo estrutural, os autores destacaram a SDS como um instrumento de medida com mais vantagens, por ser conciso, eficiente e suficiente para avaliar as incapacidades ou prejuízos associados aos transtornos de ansiedade, comumente avaliados por instrumentos longos ou muito detalhados, como a SAS-S e o MOS.

Ressaltaram que as dimensões avaliadas por esses instrumentos não foram suficientes para avaliar com especificidade pessoas com TAS, visto que estas mostram mais dificuldades nas relações e nas atividades fora do trabalho quando comparadas às pessoas com transtorno de pânico e obsessivo-compulsivo, apontando para a necessidade de instrumentos que incluam aspectos específicos para a avaliação dessas características. Desse modo, ressaltaram a relevância da identificação de áreas específicas de prejuízos funcionais.

Com relação ao quarto estudo analisado ${ }^{14}$, foi investigada a consistência interna, a validade e a sensibilidade da LSRDS, da DP e da SDS, visando avaliar se os prejuízos funcionais de pessoas com TAS estão relacionados ao transtorno em si ou às conseqüências da associação com as comorbidades, principalmente a depressão. Participaram do estudo 153 pessoas com diagnóstico 
principal de TAS, selecionadas em clínicas ou centros universitários de estudo e tratamento das desordens de ansiedade, avaliadas com base nos critérios da SCID-IV e dos instrumentos LSRDS, DP, SDS e BDI. Adotaram bons cuidados metodológicos, como a randomização para a seleção da amostra, o uso de avaliadores experientes e cegos em relação aos diagnósticos e, ainda, a avaliação por meio de instrumentos já aferidos e com boas qualidades psicométricas demonstradas em estudos prévios. Os autores destacaram que a LSRDS, a DP e a SDS mostraram boa capacidade de mensuração dos prejuízos funcionais relacionados ao TAS. No entanto, em relação à sensibilidade, a LSRDS e a DP foram mais eficientes para detectar os indicadores de prejuízos associados à ansiedade social.

Na avaliação psicométrica dos instrumentos, relataram que a consistência interna da LSRDS mostrou "nas duas últimas semanas" e no "pior momento ao longo da vida" alfa de Cronbach de 0,75 e 0,82 respectivamente; a DP apresentou alfa de Cronbach de 0,71 para as categorias "nas duas últimas semanas" e 0,79 para "pior momento ao longo da vida". Quanto à SDS, a consistência interna foi menor: mostrou alfa de Cronbach de 0,55. Foi avaliada a correlação dos itens com o escore total para cada escala, objetivando investigar a contribuição de cada item. Nesta avaliação, a LSRDS variou de 0,38 a 0,65 para a categoria "nas duas últimas semanas" e 0,41 a 0,62 para a categoria "pior momento ao longo da vida"; a DP mostrou variação de 0,22 a 0,62 para a avaliação "nas duas últimas semanas" e de 0,32 a 0,62 para "pior momento ao longo da vida". A variação da correlação da SDS foi de 0,27 a 0,51.

Os autores ressaltaram que as medidas de prejuízos funcionais apresentaram alta correlação, sendo que a LSRDS e a DP apresentaram maior correlação com o escore total da SDS do que com os domínios trabalho e incapacidade social. Constataram, ainda, que as medidas de prejuízos funcionais foram fortemente relacionadas às medidas de ansiedade social, de depressão e de qualidade de vida.

O estudo das diferenças de prejuízos em função da classificação do TAS e dos aspectos sociodemográficos mostrou, em concordância com a literatura, que indivíduos que preencheram os critérios para o subtipo generalizado apresentam escores mais altos de prejuízos funcionais quando comparados ao tipo não-generalizado. De modo semelhante, as pessoas solteiras ou separadas e com menor índice de escolaridade apresentaram mais prejuízos em comparação com as pessoas casadas e com maior escolaridade. Quanto à etnia e ao gênero, não foram observadas diferenças estatisticamente significativas.

Com base na análise de regressão, relataram que a depressão foi a variável de maior contribuição para o aumento dos prejuízos funcionais; no entanto, observaram que mesmo após o controle dessa variável, as medidas de prejuízo ainda apresentaram variância estatisticamente significativa, sugerindo que as dificuldades nas atividades que sustentam o cotidiano são relacionadas ao TAS e não decorrem das comorbidades comuns a esse transtorno.

Quanto à associação entre os níveis de prejuízos funcionais e a gravidade dos sintomas, os autores relatam que o nível de prejuízo muda em relação à remissão do sintoma. Ressaltaram que a avaliação dos prejuízos funcionais é mais que a ênfase na vivência pessoal de vários sintomas, tendo como foco a identificação do modo como a desordem interfere nas ações cotidianas do indivíduo, o que guarda relação com variáveis demográficas e clínicas.

Consideraram que as medidas de prejuízo funcional são um importante determinante das necessidades do paciente e da efetividade dos procedimentos de intervenção.

Nos quatro estudos analisados, observou-se uma ampla variedade de instrumentos, porém em concordância com Schneier et. al. ${ }^{10}$, Mattick \& Clarke $^{15}$, Quilty et. al. ${ }^{13}$ e Hambrick et. al. ${ }^{14}$, ressalta-se a necessidade de instrumentos validados e específicos para a avaliação dos prejuízos funcionais relacionadas ao TAS. Dentre os instrumentos mais usados para tal avaliação, destacase a SDS, desenvolvida para avaliar a associação entre a gravidade dos sintomas psiquiátricos e a incapacidade social. Esta escala, apesar de não ser um instrumento para avaliação específica dos prejuízos associados ao TAS, tem sido amplamente usada para esta finalidade.

Nesse sentido, dadas as boas qualidades psicométricas demonstradas, ressalta-se a relevância da LSRDS e da DP como instrumentos específicos e de referência para a avaliação dos prejuízos 


\section{ESTUDOS RELACI ONADOS AOS PREJ UÍ ZOS FUNCI ONAIS E À QUALI DADE DE VI DA DE PESSOAS COM TAS}

Foram incluídos e analisados, neste conjunto, seis estudos $5-7,16-18$. Dentre estes estudos, quatro avaliam a qualidade de vida de forma geral ${ }^{5-7,16}$, e dois tratam de domínios específicos relacionados à qualidade de vida: o econômico ${ }^{17}$ e o escolar ${ }^{18}$.

Em relação aos delineamentos, as amostras variaram de 44 a 8.537 sujeitos (mediana $=4.290$ ) de ambos os sexos, com idade variando de 15 a 65 anos, provenientes de amostras clínicas e da população geral. Os participantes incluídos nas amostras foram avaliados por meio de entrevistas estruturadas, baseadas nos manuais diagnósticos DSM-III-R, DSM-IV e Classificação Estatística Internacional de Doenças (CID-10), a saber: ADIS-IV-L, SCID, Composite International Diagnostic Interview (CIDI) e Clinical Interview Schedule-Reviewed (CIS-R). Cinco estudos adotaram como critério de exclusão a presença de outros transtornos psiquiátricos, e um estudo incluiu nos critérios de exclusão a presença de TAS subclínico e de comorbidades comumente associadas ao transtorno, como a distimia, transtornos alimentares e uso ou abuso de substâncias.

Quanto à qualidade de vida de pessoas com transtornos de ansiedade ou especificamente com TAS, os seis estudos constataram prejuízos e insatisfação com a vida e com a saúde. As características dos delineamentos e as especificidades dos estudos serão abordadas a seguir.

No primeiro estudo analisado neste conjunto ${ }^{16}$, foi estudada a influência do TAS na qualidade de vida, comparando pessoas com TAS a pessoas sem transtornos psiquiátricos, visando avaliar o impacto econômico do TAS com base nos índices de produtividade e de uso de serviços de saúde. Para tanto, foram avaliadas 130 pessoas da comunidade, sendo 65 pessoas com diagnóstico exclusivo de TAS e outras 65 pessoas que não preencheram critérios para TAS ou para outros transtornos psiquiátricos emparelhados quanto ao sexo e idade. Foram adotados como critérios de exclusão a presença de transtornos psiquiátricos prévios ou atuais, comorbidades comumente associadas ao TAS e TAS subclínico, completando, assim, o que os autores chamaram de grupo de pessoas com TAS puro ou não complicado.

Foram usados como instrumentos o Social Functioning (SF-36) para a avaliação da qualidade de vida geral, a LSRDS para avaliação de prejuízos funcionais e incapacidades associadas ao TAS, o Reilly Work Productivity and Impairment Questionnaire (WPAI) para mensurar a produtividade no trabalho e o Health Service Use Inventory (HSUI) para a informação sobre o uso de serviços de saúde ao longo da vida.

O estudo mostrou que o TAS afeta quase todos os aspectos da vida do indivíduo, diminuindo significativamente a qualidade de vida, principalmente nas áreas do desempenho escolar, autocuidado e relações afetivas. Em relação à produtividade no trabalho, os autores destacaram que este se mostrou fortemente comprometido quando comparado ao grupo de pessoas sem TAS. Foram significativamente mais freqüentes no grupo de pessoas com TAS o desemprego, o absenteísmo e os prejuízos no desempenho profissional. As atividades de autocuidado também se mostraram comprometidas e associadas com a diminuição da qualidade de vida. 
algum tipo de assistência ao longo da vida, sendo que as intervenções farmacológicas foram as mais freqüentes. Destaca-se, nesse estudo, a presença de indicadores de funcionalidade relativos ao desempenho profissional, interações sociais e autocuidado, além da associação com prejuízos na qualidade de vida.

O segundo estudo 5 avaliou: a) o impacto do TAS na autopercepção da qualidade de vida; b) a relação entre a qualidade de vida e a gravidade dos sintomas; c) a variação da qualidade de vida frente aos subtipos do TAS e a associação a transtorno de personalidade evitativo; e; d) a efetividade da terapia cognitivo-comportamental (TCC). Participaram desse estudo 44 pessoas com diagnóstico de TAS, avaliadas de forma sistemática, adotando como critério de exclusão transtorno bipolar, transtorno psicótico ou dependência de substância psicoativa. Para a avaliação dos participantes, utilizaram as entrevistas estruturadas do DSM-III-R e do DSM-IV e os instrumentos de avaliação: QOL, SAS, SIAS, Social Phobic Scale (SPS), Personality Disorders Examination -DSM-III-R, BDI, STAI e Fear Questionnaire (FQ). Todos os sujeitos do estudo foram tratados em grupo, tendo como abordagem terapêutica a TCC, sendo avaliados no momento anterior e posterior a essa intervenção por avaliadores independentes e cegos em relação ao diagnóstico dos participantes.

Os autores ressaltaram que as pessoas com TAS avaliaram sua qualidade de vida como significativamente pior quando comparadas a uma amostra de pessoas sem transtornos psiquiátricos com características demográficas semelhantes. Observaram também que a medida de qualidade de vida variou em relação aos subtipos do TAS, ao transtorno de personalidade evitativo e ao estado civil, sendo que o grupo com diagnóstico comórbido de TAS generalizado e de transtorno de personalidade evitativo apresentou os piores escores de percepção da qualidade de vida, expressos por dificuldades nas relações afetivas, alterações de humor e maior gravidade dos sintomas.

Em relação à percepção da qualidade de vida antes e após o tratamento com TCC, o estudo mostrou que o escore da medida de qualidade de vida avaliada por instrumentos foi significativamente maior após o tratamento, mostrando não somente a melhora do sofrimento relacionado aos sintomas, mas também da percepção de melhora da qualidade de vida geral. Os indicadores de prejuízos funcionais foram relacionados nesse estudo a aspectos demográficos e clínicos, além de terem sido sensíveis a intervenção terapêutica.

O terceiro estudo analisado ${ }^{7}$ avaliou as dificuldades psicossociais e funcionais, os prejuízos e o impacto do TAS para a qualidade de vida geral dos portadores. Avaliaram 150 pessoas provenientes de amostra da comunidade, sendo $65 \mathrm{com}$ diagnóstico de TAS sem comorbidades, 51 com TAS associado a comorbidades e 34 pessoas que não preencheram todos os critérios para o diagnóstico de TAS, incluídas no grupo TAS subclínico, objetivando relacionar o desempenho do grupo de TAS sem comorbidades ao de um grupo de pessoas com doença de curso flutuante e crônico, a saber, 65 pessoas com história de infecção por herpes, que não preencheram critérios para o TAS ou para outros transtornos psiquiátricos, emparelhadas quanto a idade e sexo. Para a inclusão nas amostras, os participantes foram avaliados por entrevistadores experientes e devidamente treinados para o uso da CIDI e dos outros instrumentos usados no estudo: SF-36, LSRDS, WPAI e HSUI.

Com base nos resultados, encontraram que o grupo de portadores de TAS sem comorbidade apresentou indicadores de significativo prejuízo na qualidade de vida nos domínios relacionados à saúde geral, ao funcionamento social, à vitalidade e à saúde mental. As pessoas com TAS provenientes do grupo subclínico mostraram resultados parecidos ao do grupo de pessoas com TAS sem comorbidades, enquanto que para o grupo de TAS com comorbidades os níveis de prejuízos foram acentuadamente mais graves na maioria dos domínios da SF-36 e da LSRDS.

Relataram que as pessoas com TAS apresentavam indicadores de limitações profissionais e, conseqüentemente, limitações quanto aos recursos financeiros. Destacaram que os indivíduos com TAS gastaram mais com serviços de saúde e ganharam menos referindo dificuldades em conseguir emprego e nas relações de trabalho, além de perda da produtividade. Em geral, estes prejuízos foram avaliados como piores ao longo da vida quando comparados ao momento atual, provavelmente devido ao prejuízo cumulativo que incide sobre o comportamento adaptativo dos 
participantes.

Ressaltaram que, no TAS, enquanto quadro de evolução crônica, os prejuízos funcionais comprometem significativamente a produtividade, as relações sociais e a participação nas atividades da vida cotidiana, implicando em perdas econômicas e gastos com saúde.

No quarto estudo6, foi avaliada a extensão e a amplitude dos prejuízos funcionais e na qualidade de vida de pessoas com TAS e a relação desses prejuízos com a depressão maior. Para este estudo, foram analisados os dados referentes à aplicação do Suplemento de Saúde Mental Pesquisa de Saúde de Ontário, pesquisa epidemiológica realizada para a identificação de transtornos psiquiátricos na comunidade canadense. Para tanto, foram analisados os dados de 8.116 pessoas avaliadas por meio da CIDI e separadas quanto aos subtipos do TAS e a presença de depressão maior. Para a avaliação dos prejuízos funcionais e da insatisfação nas atividades cotidianas, foram usados os itens do Suplemento de Saúde Mental, e para a avaliação da qualidade de vida, utilizaram a Quality of Well-Being Scale (QWBS).

Quanto aos achados, destacaram que as pessoas com TAS relataram maiores prejuízos funcionais, menor satisfação com a vida e menor percepção de bem-estar quando comparadas às pessoas sem o transtorno. A depressão maior e as diferenças sociodemográficas tiveram pequena contribuição para este resultado, constatando que a presença de depressão não apresentou significância estatística na relação com os domínios relativos a prejuízos nas atividades cotidianas, nas relações interpessoais e no desempenho escolar.

Ressaltaram o impacto dos prejuízos e a redução da qualidade de vida de pessoas portadoras de TAS, assim como a necessidade de ações e políticas de saúde que incluam o tratamento e a prevenção. Nesse estudo, os indicadores de prejuízos funcionais foram associados às percepções de bem-estar e satisfação com a vida.

O quinto estudo analisado ${ }^{17}$ teve como foco específico a vida profissional, as limitações na ascensão profissional quanto a ocupar cargos administrativos, manter-se empregado e a renda familiar. Os dados analisados decorreram de uma pesquisa populacional de morbidade psiquiátrica de um estudo epidemiológico amplo, com 8.501 pessoas sem transtornos psiquiátricos em comparação com 63 pessoas com TAS. Destas, 27 apresentavam comorbidades, tais como: depressão, transtorno obsessivo-compulsivo e transtornos psicóticos, avaliados por meio do CIS-R.

Constataram que as pessoas com diagnóstico de TAS experimentaram prejuízos na vida pessoal, acadêmica e profissional, além de dificuldades nos relacionamentos afetivos, sendo a maioria separada, divorciada ou solteira. As pessoas com TAS mostraram maior tendência a serem dependentes financeiramente de familiares e a estarem desempregadas. Observaram, ainda, que os níveis de prejuízos foram mais graves no grupo de TAS com comorbidade.

Os autores reforçaram que o TAS é uma condição sub-reconhecida e subdiagnosticada, o que favorece a cronificação e o maior uso dos serviços de saúde, destacando a necessidade de cuidados preventivos e de tratamentos psicoterápicos e psicofarmacológicos mais efetivos. Destaca-se, nesse estudo, a presença de indicadores associados a condições demográficas e a associação a prejuízos econômicos.

O sexto estudo analisado ${ }^{18}$ avaliou a associação entre abandono escolar e presença de TAS, tendo como foco a avaliação específica da vida acadêmica de 201 pessoas com diagnóstico de TAS sem comorbidade. Todos os participantes responderam a instrumentos de auto-avaliação, sendo avaliados quanto à presença de depressão, de prejuízos no ajustamento social e a sintomas de ansiedade por meio dos instrumentos: BDI, STAI, FQ, SAS e SDS.

Constataram que, para $48,8 \%$ dos indivíduos, foi identificada a associação entre abandono escolar prematuro e a presença de indicadores de ansiedade. Dentre estas pessoas, $61,2 \%$ apresentavam histórico de TAS recente ou ao longo da vida. 
As avaliações mostraram que as razões mais freqüentes para o abandono escolar estavam relacionadas a: sentir-se nervoso em sala de aula ou na escola, necessidade de trabalhar para ser independente, perda de interesse pela escola, temor de falar na frente dos colegas de classe, timidez frente ao professor e abuso de álcool ou drogas. O estudo mostrou, ainda, que 34,8\% dos participantes referiram que as preocupações e a ansiedade motivavam atrasos e os mantinham em casa, impedindo-os de freqüentar a escola.

Os autores destacaram que os transtornos de ansiedade, especialmente o TAS generalizado, foram fortemente associados ao abandono precoce da escola, evidenciando a necessidade de diagnóstico preciso e tratamento efetivo, além da necessidade de identificação dos indicadores de prejuízos de modo a planejar e aplicar práticas de saúde mental. Os indicadores de prejuízos funcionais nesse estudo destacaram a vida acadêmica e o falar em público.

Ao analisar esses estudos, constata-se que, ao caracterizar os prejuízos funcionais, eles contribuem para o conhecimento do impacto do TAS para a vida dos portadores, referendando uma visão abrangente de saúde como um estado de bem-estar físico, mental e social, que pode ser avaliado por meio da satisfação com a vida, da percepção de bem-estar e da capacidade de desempenhar os afazeres cotidianos, e não somente pela avaliação da diminuição ou ausência de sintomas.

Os estudos analisados mostraram que a percepção de insatisfação com a vida e as dificuldades apresentadas pelas pessoas com TAS estão fortemente relacionadas a prejuízos funcionais que incidem no desempenho acadêmico, na produtividade, no trabalho, nos relacionamentos sociais, familiares e afetivos, o que por sua vez tem uma associação com prejuízos econômicos e com o uso de serviços de saúde. Tais dificuldades caracterizam comprometimento na participação e no engajamento em atividades essenciais da vida cotidiana.

\section{A avaliação dos prejuízos funcionais e as implicações para as práticas de saúde}

Os estudos sobre a $\mathrm{ClF}^{19,20}$ ressaltam a importância, para os profissionais da saúde, de estudos sistemáticos que avaliem a funcionalidade, tendo em vista que somente o conhecimento das causas e das freqüências das doenças na população não é suficiente para as intervenções, ressaltando a necessidade da identificação do que as pessoas fazem ou não podem fazer na sua vida diária como recurso que instrumente as práticas da saúde mental.

Em relação à avaliação específica dos prejuízos funcionais relacionados ao TAS, a análise da literatura recente aponta para a disponibilidade de instrumentos que avaliam este construto, aferidos para outros países, assim como o impacto dos prejuízos funcionais na participação cotidiana.

Como demonstrado neste estudo de revisão, destacam-se a LSRDS e a DP pelas boas qualidades psicométricas, sendo eficazes na avaliação das limitações no funcionamento cotidiano relacionadas ao TAS.

Faz-se necessário que instrumentos com tais características sejam aferidos para o nosso meio, atendendo a critérios rigorosos de tradução, adaptação, seguido de estudos de validade e confiabilidade.

O uso de medidas objetivas de identificação dos prejuízos funcionais com relação às pessoas com TAS poderá sistematizar as práticas multidisciplinares de saúde mental, favorecendo a avaliação, o planejamento e a intervenção, incluindo como foco a participação nas atividades cotidianas $^{10}$.

Dentre as práticas multidisciplinares de saúde mental, destaca-se a relevância de tais estudos para a terapia ocupacional, considerando que os prejuízos funcionais, como as dificuldades na participação em atividades profissionais, sociais, de lazer e de autocuidado, assim como as restrições próprias de um cotidiano empobrecido, constituem-se o foco principal de estudo e das intervenções dessa área ${ }^{21}$. 


\section{CONCLUSÃO}

Entre os estudos analisados, a maioria utilizou a LSRDS e a DP como instrumentos de referência para avaliar o impacto do TAS nas ocupações diárias de populações clínicas em comparação a populações não-clínicas, com as mesmas características sociodemográficas. Esses instrumentos foram aferidos e demonstraram boas qualidades psicométricas no que se refere ao estudo da consistência interna, da validade concorrente, da correlação dos itens com o escore total, da análise correlacional com outros instrumentos ou da identificação dos seus principais componentes.

Tendo em vista a relevância e a contribuição desses instrumentos para a avaliação dos indicadores de prejuízos funcionais, considera-se necessários estudos empíricos nacionais que possibilitem a utilização sistemática dos mesmos. Tal recurso metodológico poderá instrumentar intervenções no contexto das práticas multidisciplinares de saúde mental, inclusive as da terapia ocupacional.

\section{REFERÊNCIAS}

1. Brunello N, den Boer JA, Judd LL, Kasper S, Kelsey JE, Lader M, et al. Social phobia: diagnosis and epidemiology, neurobiology and pharmacology, comorbidity and treatment. J Affect Disord. 2000;60(1):61-74.

2. Furmark T. Social phobia. From epidemiology to brain function. Acta Universitatis Uppsaliensis. Comprehensive summaries of Uppsala dissertations from the Faculty of Social Sciencies 97. Uppsala: Uppsala Universitet, Tryck \& Medier; 2000.

3. Kessler RC. The impairment caused by social phobia in the general population: implications for intervention. Acta Psychiatr Scand Suppl. 2003; (417): 19-27.

4. Lecrubier Y, Wittchen HU, Faravelli C, Bobes J, Patel A, Knapp M. A European perspective on social anxiety disorder. Eur Psychiatry. 2000; 15(1):5-16.

5. Safren SA, Heinberg RG, Brown EJ, Hoole C. Quality of life in social phobia. Depress Anxiety. 1996-1997; 4(3): 126-33.

6. Stein MB, Kean YM. Disability and quality of life in social phobia: epidemiologic findings. Am J Psychiatry. 2000; 157(10): 1606-13.

7. Wittchen HU, Fuetsch M, Sonntag H, Müller N, Liebowitz M. Disability and quality of life in 
pure and comorbid social phobia. Findings from a controlled study. Eur Psychiatry. 2000; 15 (1): 46-58.

8. Manual Diagnóstico e Estatístico de Transtornos Mentais. Texto revisado DSM-IV-TR. Porto Alegre: APA-Artmed; 2002.

9. Wittchen HU, Stein MB, Kessler RC. Social fears and social phobia in a community sample of adolescents and young adults: prevalence risk factors and co-morbidity. Psychol Med. 1999;29 (2): 309-23.

10. Schneier FR, Heckelman LR, Garfinkel R, Campeas R, Fallon BA, Gitow A, et al. Functional impairment in social phobia. J Clin Psychiatry. 1994;55(8):322-31.

11. Hambrick JP, Turk CL, Heimberg RG, Schneier FR, Liebowitz MR. The experience of disability and quality of life in social anxiety disorder. Depress Anxiety. 2003; 18(1):46-50.

12. Organização Mundial da Saúde (OMS). CIF: Classificação internacional de funcionalidade, incapacidade e saúde da organização mundial da saúde. Centro colaborador da Organização Mundial da Saúde para a família de classificações internacionais, org. São Paulo: EDUSP; 2003.

13. Quilty LC, Van Ameringen M, Mancini C, Oakman J, Farvolden P. Quality of life and the anxiety disorders. J Anxiety Disord. 2003; 17(4):405-26.

14. Hambrick JP, Turk CL, Heimberg RG, Schneier FR, Liebowitz MR. Psychometric properties of disability measures among patients with social anxiety disorder. J Anxiety Disord. 2004; 18 (6): 825-39.

15. Mattick RP, Clarcke JC. Development and validation of measures of social phobia scrutiny fear and social interaction anxiety. Behav Res Ther. 1998;36(4):455-70.

16. Wittchen HU, Beloch E. The impact of social phobia on quality of life. Int Clin Psychopharmacol. 1996;11 Suppl 3:15-23.

17. Patel A, Knapp M, Henderson J, Baldwin D. The economic consequences of social phobia. J Affect Disord. 2002;68(2-3):221-33.

18. Van Amerigen M, Mancini C, Farvolden P. The impact of anxiety disorders on educational achievement. J Anxiety Disord. 2003; 17(5):561-71.

19. Farias N, Buchalla CM. A classificação internacional de funcionalidade, incapacidade e saúde da organização mundial da saúde: conceitos, usos e perspectivas. Rev Bras Epidemiol. 2005;8 (2): 187-93.

20. Nubila HBV. Aplicação das classificações CID-10 e CIF nas definições de deficiências e incapacidade [tese]. São Paulo: Universidade de São Paulo; 2007.

21. Benetton MJ, Tedesco SA, Ferrari SML. Hábitos, cotidiano e terapia ocupacional. Rev Cent Estud Ter Ocup. 2003; 8(8):27-40.

\section{论 Correspondência}

Luciene Vaccaro de Morais

Departamento de Neurologia, Psiquiatria e Psicologia Médica, Faculdade de Medicina de Ribeirão Preto, USP, Hospital das Clínicas, 3o andar 
Av. Bandeirantes, 3900

CEP 14049-900, Ribeirão Preto, SP

Tel.: (16) 3602.2837, Fax: (16) 3602.2544

E-mail: Ivaccaro@usp.br, luciene_vaccaro@terra.com.br

Recebido em 12/05/2007.

Aceito em 06/07/2007.

* Este trabalho foi realizado no Departamento de Neurologia, Psiquiatria e Psicologia Médica da Faculdade de Medicina de Ribeirão Preto, USP. Comunicação oral e resumo baseados na revisão foram apresentados no X Congresso Brasileiro de Terapia Ocupacional, realizado em Goiânia, em 18 de maio de 2007 . 